\title{
Investigating Pay-as-you-go to address issues of trust, privacy and security around media use at home.
}

\author{
Emmanuel Tsekleves $^{1}$, Roger Whitham ${ }^{2}$, Koko Kondo ${ }^{3}$, Annette Hill ${ }^{3}$ \\ ${ }^{1}$ Brunel University, Kingston Lane, Uxbridge, UB8 3UE, UK \\ ${ }^{2}$ Lancaster University, Lancaster, LA1 4YW, UK \\ ${ }^{3}$ University of Westminster, Northwick Park, Harrow, HA1 3TP, UK \\ emmanuel.tsekleves@brunel.ac.uk, r.whitham@lancaster.ac.uk, k.kondo@wmin.ac.uk, \\ a.hill@wmin.ac.uk
}

Tel: 0044-(0)1895-267379

\begin{abstract}
: in this paper we report on a study conducted in 2007 and 2008 looking at the media use habits of 27 families in the Greater London area. The project builds on previous work studying media use within a similar group in 2006. The study investigated attitudes towards the issues of trust, privacy and security, across different types of media and particularly the Television (TV) within the home environment. We explored the use of a Pay-as-you-go (PAYG) concept as a means of addressing issues of trust, privacy, billing and security around media in the home. To facilitate the study we rapidly prototyped an experimental home media device and asked participants to use and respond to it. Our key findings show the desirability of devices and services that incorporate a payment system which would help in regulating spending and allow household members to manage their own media purchases. The PAYG concept was well received by study participants as a means to prevent unauthorised spending and help manage costs. Participants were also enthusiastic about more transparent billing mechanisms and the possibility of monitoring the TV and media use of younger household members.
\end{abstract}

Key words: Interactive Television, user studies, trust, privacy, security, usercentric media, media use in the home.

\section{INTRODUCTION}

Media consumption within the home has seen substantial change in recent years. New forms of media and associated devices have become prevalent in domestic settings, altering the types of entertainment and information-centric activities individuals engage in. With increasingly general forms of media [1], such as the 
World Wide Web, come increasingly complex systems and the risk of an accompanying increase in the difficultly of using them. In addition, there has recently been a dramatic increase in the use of portable, high-quality, web-enabled screens in the home such as the Apple iPhone, iPad and other android-based smart phones and tablets.

Our research is focused around Television (TV) as a common denominator of entertainment and information access within the home. A family TV typically offers a high quality audio and visual experience compared with other devices in the home. It is also typically situated prominently in a shared social space, such as the living room or kitchen. These common features of TV usage make for a device all members of the family can understand, situated in an environment suitable for shared consumption of media. In this paper we focus on the security, trust and privacy issues surrounding the use of TV and interactive Television (iTV) within the modern household with the aim of improving applications and services available in the home.

After considering related literature, this paper describes our study in two distinct parts. Firstly we describe the process of engaging with study participants and finding out about their current concerns and issues with regards to trust, privacy and security. After reporting on these results we then describe how these findings drove the design of an experimental second screen device which was presented to participants as a point of discussion in the final stages of the study.

\section{RELATED WORK}

\subsection{The Core TV User Experience}

TV is often seen as being at the epicentre of the household media consumption. It is principally an entertainment activity and part of leisure experience in the domestic environment [2-6]. It has a prominent place in the household, especially when taking into account ageing populations and their reliance on such household technologies for leisure and social activities [7].

Additionally, TV is regarded as a lean-back medium with its users commonly reporting a relaxing and passive attitude towards it [8]. TV has been also the centre point of the entertainment experience with all or most household members 
making regular, if not always shared, use of it. Geerts et al. have conducted extensive research on the subject of TV and sociability. They have published findings on TV programme genres that promote social interaction by watching TV together and over distances $[5,9]$.

The TV is also often seen as comfortable and familiar and it has been traditionally characterized as a trusted medium [10]. As such it can be perceived as a less intimidating technology to individuals less used to interacting with digital media, especially when compared to modern day Personal Computers (PCs) [11]. For elderly people the burden of accessing the Internet through the TV is lower than buying a PC and learning to interact with it [5]. Trends towards simpler and easier to use services are also embodied in the current generation of portable touchscreen devices, such as the Apple iPad.

These notions of the core TV user experience have, however, been altered by the advent of iTV in the home environment, increasing even further the role that Television plays for many and raising some very important issues related to the impact this new form of TV medium has on people’s lives.

Interactivity now enables a traditionally passive medium to support a two-way interactive experience. However, several studies have shown that interactivity in the TV domain is not always welcomed by viewers, and in some cases, may be disruptive to the TV user experience [12-15]. This is especially likely as iTV services attempt to transform the TV from a lean-back to a lean-forward medium. The level of interactivity is not only limited by the potential of the technology, namely display mechanisms, hardware and interaction models [16-17], but also by the user's willingness to interact [5]. It has been found though that the TV user experience benefits more from an optional interaction model, where the user decides how much interaction they wish to engage in, if any $[12,18]$

In this context it is also interesting to note that although TV is usually controlled by a single person, it is clearly a medium that supports social activities in the domestic setting $[4,12,19]$.

The aforementioned changes in the TV and media use landscape in the home raise a number of issues and concerns with regards to trust, privacy and security. 


\subsection{Television and Trust}

Trust in a technology or service is of utmost importance as it relates to the user's reliance on consuming a service and its content as well as the user confidence in managing that service [20]. Usually trust is associated with familiarity and recognisability [21] and in the case of the traditional TV both of these user acceptance factors are very important. Several studies have shown that TV is positively associated with familiarity and trust [10, 12, 22]. Some studies, however, suggest that with the advent of iTV, user perception is starting to change.

Negative user experiences of a service or technology are usually a key factor for loss of trust [23]. Kaasinen et al in their TV study [20] found that mistrust was the main negative factor in user acceptance due to coverage and technical issues. Berglund also indicated the importance that trusting the content as well as the technology has for many users [6]. Although his study was based on the use of the Internet, it is still relevant that user mistrust of information provided by particular web sites created a perception of mistrust to the Internet media as a whole. In the same respect we suggest that user mistrust on a specific programme/service provider (TV channel) may result in mistrust of other TV programmes/services of the same broadcaster.

As already discussed, TV is seen as a social activity that offers its users a shared experience. Bernhaupt et al. define this shared experience as the seamless blend of user experience of products and social interaction [4]. Thus within this shared experience context trust becomes very significant since the sense of unity in the social group depends not only on people's common interests but also trust with one another [24].

Although the literature emphasizes the importance of trust for interactive Television there is little work published on developing trust in iTV. There has been extensive work in past years on developing trust in another interactive household medium, that of the Internet and more precisely e-commerce [25-27].

\subsection{Television and Privacy}

People see their home as a personal and social space at the same time, often associating it with relaxation and a strong feeling of privacy. Nowadays the home 
is filled and associated with media technology employed for both individual (e.g. Personal Computer, mobile phone) and shared household use (e.g. Television). Privacy is an important issue that needs to be considered when addressing shared use of media technology [4]. People do not want other household members to observe what they are currently viewing, a finding Buchinger et al. report to be particularly strong in Belgium and the UK [28].

Support for privacy is more readily incorporated into technologies such as PCs and mobile phones that typically assume a single user or owner. However in the case of Television and specifically iTV privacy issues can be more difficult to address due to the fact that the TV is typically located in a shared household space and accommodates both private and group media use (e.g. watching a publicly broadcasted TV programme as opposed to a DVD/Blu-ray movie or personal video recording).

Several studies have been conducted within this domain looking in particular at interactive TV and have produced very different findings. Bernhaupt et al. in their ethnographic study in Austria found that users do not associate typical privacy concerns with iTV [29]. In fact some of the household members reported that TV was the only device in the household that did not cause concerns about privacy. Cesar et al. on the other hand indicate that there are privacy concerns for media sharing in the iTV domain; whereas sharing content with other people outside home was seen as a value-added service, the participants did not find appealing to share the content within the home [30]. In addition to this Geerts et al. found that privacy is a key factor for the acceptance and use of this type of social Television service [5]. In fact Geerts and De Grooff provide a set of heuristics for social Televison in which they emphasize the importance of ensuring both personal privacy and group privacy when designing a social TV service [31]. Another interesting project investigating the subject of iTV and privacy is the EU FP7 project Together Anywhere Together Anytime (TA2). In this project it was found that all of the participating parents, if they shared media, they would do so via communication methods they perceived as private, such as email, via files on CDs or DVDs [32].

It therefore seems that the balance between privacy and personalization features becomes a major issue especially as iTV becomes more widespread [33]. 


\subsection{Television and Personalisation}

Mobile phones are regarded by users as private media devices mainly due to the fact that they have a single user. Computers are also considered as private media devices despite having more than one user [4]. The main reason behind this is that they are designed to support individual, solitary, stationary use, where users can operate the same PC device but still maintain their privacy and private data via the use of personal profiles. These personal profiles usually employ a password that enables each individual to access their own data and prevents them from accessing other users' data through the same shared device. The use of profiles is well established and spread on Internet applications and particularly social networking websites where users can personalise their preferences [34].

Following the success of user profiles in the PC and Internet domain several projects and studies have investigated their potential use in the TV and iTV environment. Two studies in Austria [35-36] investigated the personalisation of iTV services via profiles, finding that it was well accepted by the participants. They also investigated the use of a fingerprint reader instead of the conventional alphanumeric code as a new means of accessing profiles and increasing security, which according to [35] was perceived positively by families for the most part. In addition to the aforementioned studies, Vlachogiannis et al. [37] have also investigated the use of profiling for iTV using the MPEG-21 standard and Luyten et al. [38] have made use of profiles in their Telebudies social TV system.

Lastly Egelman et al. found evidence that current user account models are not well suited for the domestic environment [39]. For this reason they developed Family Accounts, a new user account model for shared home computers that offers a compromise between a single shared profile and individual profiles for each family member. Navarro et al. also emphasize the need of focusing on family profiles [40].

This family profile model resulted in positive user responses but was aimed at and tested with shared family computers, not iTV. It would be therefore interesting to adopt the profile model for the iTV environment and seek user feedback. 


\subsection{Security and Television}

Within the TV context, security is seen as an important concern in the development of new interactive media services for the home. Several user studies have found that this is especially true in households with young children, where parents seek ways of gaining better control over their children viewing habits [4, 26].

More precisely Keeling et al. found that $73 \%$ of participants in their study were concerned about what their kids might be able to view and buy on the TV [19]. Bernhaupt et al. found that whenever security and personalization are addressed, the user's habits and use patterns have to carefully be taken into account [4]. Another ethnographic study [41] found that security concerns varied enormously from household to household, with those that contained adults alone wanting something to prevent intruders from gaining access to the system from their home. Households with young children wanted to protect them from media and video game content unsuitable for their age. In another study [32] parents expressed security concerns about who has access to their information and especially their media, with a single parent leading or controlling the adoption of technology and media. It was also established that there was a demand for an application that allows a parent (termed gatekeeper/administrator by [32]) to be in control of the media consumption of their children. As Bernhaupt et al. indicate security for the TV is mainly associated with child safety such as reducing the access of children to TV content [29]. With respect to this Wilfinger et al. suggest the use of profiles that parents set in order to control what their children watch on TV, especially when the parents are engaged with other household activities [35].

Younger members of the household, despite their frequent reliance on PCs and the Internet for entertainment purposes, also make use of the TV as or in support of social activities, though their parents often have first say in what is watched [42]. Younger members are also very open to the use of online social networking activities, whilst parents, on the other hand, have reservations towards these [32].

Another important security issue that relates to iTV in particular is about payments made through the TV. This is not very well documented in the literature but it is a concern that becomes apparent as iTV use becomes more widespread. Especially given the social aspects of TV use, security features are difficult to get right. The social context of this medium's use has implications for privacy, 
rendering it more difficult for users to carry out certain activities, such as financial transactions, in front of other household members. With reference to this, Keeling et al. found in their study that $56 \%$ of their participants considered privacy as a key factor for security [19]. Participants in this study did not want other people in the living area to look at what they were buying and feared that people could see or hear their payment details.

Lastly there are a number of papers which place an emphasis on the user interface of the iTV application/service as a means to reinforcing security and reinforcing trustworthiness [27]. Views on this are split, as [43] highlights the contradictory relation between usability and security in that the more secure an interface is, the less usable it becomes and the more usable it becomes, the less secure it is. [44] on the other hand emphasizes the importance of visual feedback as a means of communicating safety and trustworthiness back to users with regards to any transaction based actions.

\subsection{Pay-as-you-Go and Television}

Computers are generally perceived by people as more secure compared to TV systems with users being more likely to carry out financial transactions [8]. This was shown to be based not only on the solitary nature of the PC use, but also on the fact that in a computer environment a user can retain his/her privacy by using a login profile facility [4].

Current trends in other media related technologies, such as mobile phones, illustrate the success of the pay-as-you-go (PAYG) concept from both usability and commercial perspectives. PAYG systems can be defined as those which allow the prepay of credit into an account which then acts as a limit on that account until more prepaid funds are added. PAYG systems have become popular in recent times as well as an increase in support for low-value, high-volume transactions of text, music, video and other digital media [11, 45-47]. According to Grinter and Eldridge [48] it was the introduction of the "pay-as-you-go" concept in mobile phone plans that led to increased SMS use in the UK, making it possible for teenagers to own their own mobiles. But also as Kurniawan et al. [49] indicate, that PAYG systems have been welcomed by older users, as their study revealed that particularly in the mobile phone market participants were split almost equally 
on the pay scheme (pay-as-you-go vs. pay monthly). Within this context Hui et al. have proposed a novel mobile application known as "Shair" that allows mobile users on contracts to sell their unused minutes and text messages to pre-pay customers when, e.g., they are running out of pre-paid credit [50].

Apart from the mobile phone domain, the PAYG concept has been welcomed in other digital environments. Amazon.com offers a commodity-priced storage utility, known as S3, which aims to provide storage as a low-cost, highly available service, with a simple PAYG charging model [51]. In addition to this the Transport for London has for many years been employing successfully a PAYG travel card known as the Oyster card [23]

The PAYG concept also offers benefits that are not found in other e-commerce systems, such as helping users to manage their expenses and delegate their spending more effectively, since costs are managed up-front through the purchase of vouchers [48]. It also allows the user of the services to pay according to the usage of the customer [52].

With reference to the discussion above it is interesting to explore this concept of PAYG within the context of iTV. The literature suggests that the PAYG concept has not been yet investigated within the TV domain with a limited number of studies looking into the issue of payment services through iTV. One of these studies held in Italy [53] has found that that the concept of bill payment through iTV is appreciated by users, but the actual procedure for service use can be still cumbersome, in comparison to the other channels used for payments such as the post office or the Internet.

\section{STUDY METHODOLOGY}

In our primary study, on which this paper reports, 27 households from the Greater London area participated, each with access to digital TV and broadband. This provided a total of 70 people aged $8-50+$, containing a balance of gender and socio-economic status (ABC1, C2DE - ABC1 refers to participant profiles of low to low-middle class income and C2DE to participant profiles of middle to uppermiddle socio-economic status) [54]. Table 1 provides an overview of the participants in the study. 
The study involved a total of four visits to participants' homes by researchers. The first visit took place between February and August 2007. This included 10 households who had previously participated in a previous phase of the project (this is reported in detail in [14]) in 2006 along with 17 new households. The aim of the first visit was to establish trust between the participants and the researchers and to gather information about general media use in the households. A second visit took place between August to October 2007. It included all 27 households and aimed at gathering information on participants' likes and dislikes, preferences and experiences with respect to media in the home. A third visit was carried out between November 2007 to January 2008. This visit aimed at collecting information on participant's use of media devices, media content, media device personalisation and multiplatform media.

Table 1. Overview of participating people in the study

\begin{tabular}{|c|c|c|c|c|c|c|c|c|c|c|}
\hline \multicolumn{3}{|l|}{ Age } & \multicolumn{2}{|c|}{ Gender } & \multicolumn{3}{|c|}{ Occupation } & \multicolumn{3}{|l|}{ Income } \\
\hline Age Range & No & $\%$ & Male & Female & Range & No & $\%$ & Range & No & $\%$ \\
\hline $8-12$ & 7 & 10.0 & \multicolumn{2}{|l|}{$(46 \%)$} & A & 11 & 15.7 & \multicolumn{2}{|l|}{5000} & 4.3 \\
\hline $13-17$ & 13 & 18.6 & & & B & 5 & 7.1 & $5-10 K$ & 3 & 4.3 \\
\hline $18-25$ & 6 & 8.6 & & & C1 & 17 & 24.3 & $10-15 k$ & 3 & 4.3 \\
\hline $26-35$ & 17 & 30.0 & & & $\mathrm{C} 2$ & 8 & 11.4 & $15-20 k$ & 8 & 11.4 \\
\hline $36-45$ & 6 & 8.6 & & & D & 4 & 5.8 & $20-25 k$ & 14 & 20.0 \\
\hline \multirow[t]{5}{*}{$46+$} & 21 & 24.3 & & & \multicolumn{5}{|l|}{ students } & 10.0 \\
\hline & & & & & retired & 2 & 2.9 & $25-30 k$ & 1 & 1.4 \\
\hline & & & & & \multirow{3}{*}{\multicolumn{3}{|c|}{$E$}} & $30-35 k$ & 3 & 4.3 \\
\hline & & & & & & & & $\begin{array}{l}\text { more than } \\
35 \mathrm{k}\end{array}$ & 5 & 7.1 \\
\hline & & & & & & & & don't know & 23 & 32.9 \\
\hline Total & 70 & 100.0 & \multicolumn{2}{|c|}{$70(100 \%)$} & \multicolumn{2}{|c|}{70} & 100 & \multicolumn{2}{|c|}{70} & 100 \\
\hline
\end{tabular}

A final, fourth visit was carried out between February and March 2008. The aim of the last visit was to investigate and evaluate the concepts of interactional simplicity, customisation, privacy and security. This was facilitated with the use of an experimental home media device, developed as a response to participant observations, feedback and comments from previous visits. The device acted as a vehicle for further discussion and generation of new ideas. The ethnographic 
observations made during the four visits to participant's homes were augmented with three multiple-choice questionnaires (visit 1: general media use, visit 3: use of media devices, media content, media device customisation, visit 4: privacy and security, media customisation, media interaction and sharing). Audio from 108 interviews was recorded for later analysis.

\section{INITIAL RESULTS}

Exploring the role of the TV in the domestic context, with regards to privacy, trust and security, formed our key research priority. The feedback gathered from the participant responses informed us about user needs and directed our response in the creation of an experimental device. This paper does not provide sufficient space to report on all of the ideas we explored with participants. As such we report on some of the more interesting areas of dialogue that arose during the study.

\subsection{Privacy, Trust and Security}

Security and privacy form two of the greatest concerns of UK households with respect to interactive media, as both our previous [14] and current studies indicated. Most participant concerns relate to their experiences with TV shopping.

We found that study participants in their mid forties tended to use the shopping channels, whereas younger respondents tended to used internet shopping sites for e-bargain hunting. They mocked the shopping channels and those people who used them. Most of the respondents completed their online and TV shopping from websites and TV channels they deemed as more secure and that they were familiar with.

For instance, as one of our younger participants (18 years old) indicates, his shopping habits have shifted from physical to online shopping motivated mainly by the lower prices usually found in online shops:

“Normally on Amazon or play.com or something, I don't really go to actual shops any more. I used to, but now, I thought I will get them from a shop as novelty things, but I estimate in my mind and then the shops are £5 more expensive than Amazon, so I buy through the internet." (Alex) 
Another participant (23 years old) compares the different shopping habits between her and that of her mother's:

“Me personally, I use play.com because I buy more DVDs, stuff like that. But my mum uses amazon, she also uses stuff like the worst things on QVC she can buy [laughs]. For example, electronics, she gets them all, she sits there and watches all the shopping channels." (Patricia)

Whereas a 40 years old parent admitted watching TV shopping channels not exclusively for shopping purposes rather for entertainment:

"I watch shopping channels. QVC is quite funny. It makes me laugh, when they show something really bad, and they try to convince us, taking about 20 minutes about the product." (Trevor)

People trust some established websites (banking, shopping). But they feel that there are too many passwords. Some people write them down in their diary, or make up some simple ones which can be memorised easily. One of the participants who works in an office described how he manages his passwords, raising the contemporary issue of having to manage many passwords:

"Yeah it's funny. It's everything secured, it's sensible to do. But it's getting [back in], if you forget all. You know if you go to bank and helpline, and send you passwords by post, it's about security, just for convenience, everything is about passwords now. Automatically, you end up doing it, because we registered so many different things, 20 or 30 websites, and unless you have really good memory." (Mark)

When the discussion revolved more around the issue of privacy participants discussed extensively the use of profiles to personalise the use of their media devices. Most participants are familiar with the concept of a profile from their use of computers.

"I think we use the profile because of the computer, because you've got on Microsoft you've already got the profiles there so everybody goes to the profiles, so that's something we could all slip into quite easily” (Randy)

Other participants commented on the usefulness of extending the use of profiles in the TV domain. They discussed how this would benefit household members who 
have different interests and wish to have their own list of favourite channels, preventing younger family members from gaining access to channels that parents deem inappropriate for them as well as a means of blocking unauthorised use of pay-per-view channels.

"From a larger scale point of view I think it would be good because you then can add channels that you basically say well my children are only allowed to find these certain channels on their profile, you know certain ones are off limit so I am not letting them even go near the pay-per-view channels or adult channels, from that point of view it's wicked” (Molly)

Another household agreed that TV profiles are useful but mainly for households or families consisting of several members, with one of the participants drawing from her own experience when living in a house with many tenants.

“Profiles, I think that'd definitely be really useful, for like, maybe not for us cause we are only two, but yeah when I used to live with five other people that would probably be really good" (Janet)

In addition to the discussion above, a participant pointed out another good reason why profiles would be particularly beneficial for the TV domain. According to her previous experiences in working with visually impaired people, TV profiles would enable users with accessibility issues, such as visually impaired users, to change and adjust the colours of the TV and iTV Graphical User Interface (GUI) to make them more visible to this type of users.

"Because I work with children who are visually impaired and on the laptop you have the facility to change the background and depending what your visual impairment is some children can see better on black with yellow, yellow with black and all this sort of things, so then I think if you sort of factor that in" (Kate)

\subsection{Security: Hidden Costs and Billing}

In general our study participants associated interactive television with commercialisation, hidden costs and security risks. These form the main barriers which prevent households from using interactive media services such as interactive shopping and voting. The following participant responses illustrate 
participants' concerns in calling shopping channel help lines where most of the charges are incurred from their waiting time on the phone.

"it tends to cost you money... It is premium rate or something. You think it looks very cheap but actually you spend lots on the phone bill." (Mark)

Participants also shared with us their frustrations with the additional costs incurred when contacting customer services of TV shopping channels:

"We have bought a few stuff on QVC, they are good, but the other channels, you think, 'oh it looks like this' and then when you receive it, you find it doesn't fit. You can return it. But the problem of this is when you want to return and ring them up, it tends to cost you money... It is premium rate or something. You think it looks very cheap but actually you spend lots on the phone bill." (Roger)

Participants also admitted incurring additional charges when using phone-in quiz services as well. A young professional man who had played phone-in quiz once argued the need for the regulation of this type of iTV services:

Jonathan: "yes it was like this is over a year ago I think, I just watching it, when I was at home and eleven o'clock in the morning, and I rang up, I don't remember how many times to get through, but that the only time I tried."

Greg: "it was like 25 pounds for calls. (laugh).”

Jonathan: “(laugh), so I must have rang up 25 times...I was just bored. (laugh). but at the time...You don't know it was a con...I think Ofcom should shut have them down quite long time ago."

Also charges incurred when using voting services is another area of concern that participants commented on.

"my reason for not using the interactive red button is that is not very clear, as to what you've been charged or if you've been charged, there is no transparency so I'd rather not get myself into trouble" (Jane)

Participants shared with us their experiences with other hidden cost related to interactive media, particularly interactive games. For instance, playing a game on a children's' game channel can lead to higher telephone bills, or downloading 
songs on a mobile phone can be expensive. One teenage girl entered a competition on children's' game channel, and when she won a competition to Disneyworld, her parents found out she had to pay additional costs to accept the prize.

"She won a holiday to Florida...the problem is she gets $£ 500$ towards the holiday, but It's about $£ 800$ extra for the competition, and a week in Florida. It was toll free number so I phoned up Florida, I was rather suspicious it would be a scam." (Ryan)

It was also found that several of the children's game channels contained confusing terms and conditions, which show pages of terms, with 'only 50 Pence' in big fonts and 'per minute' in smaller letters. Many respondents were wary of using these channels, and children had learnt to play free games rather than expensive ones that were itemised on the phone bill.

There seems to be therefore a recurring general mistrust theme towards the use of iTV services and applications emanating mainly from hidden charges as well as lack of transparency. The following participant quote summarises this point:

"my reason for not using the interactive red button is that is not very clear, as to what you've been charged or if you've been charged, there is no transparency so I'd rather not get myself into trouble" (Jonathan)

Interestingly one participant provided a simple solution for making iTV services requiring additional charges, such as voting, more transparent by suggesting an acknowledgement pop-up window.

"you know when you phone in or you press the red button and you start to vote and it costs you couple of quid each time...so it'll have to ask my permission so you then got control of that” (Theresa)

Most respondents did not check their iTV service accounts via the TV, relying on monthly bills posted to them to keep track of their costs. Only one household in our study regularly checked with bills on the TV itself. The majority of households assume their monthly bills will be the same, as they have a fixed rate for a package of channels.

In addition to hidden costs, participants raised concern with regulating costs related to digital media usage such as TV services like buying films and mobile phones, as this father explains: 
"she used to have monthly rate contract, and she overused it, so now she's got credit. Every month, my bill was enormous...every time she phones someone, we have to say, 'stop it stop it'." (Stephen)

\subsection{Parental Control}

Although parental control mechanisms exist for both digital TVs and the Internet as a means of monitoring children's media use habits, the majority of study participants did not make use of them. Parental controls were mainly used by households with internet access and younger children. Older teenagers were given more freedom in their access to websites and some of them knew how to disable the parental control features, or security measures. Some parents deliberately used technical problems in order to block access to sites. For example this mother explains:

"I haven't set it up for her because I don't want her to go onto the chatroom. I don't think, I don't trust her...Once she is let to do it, and she wants to look into different chatrooms, I lose control. So I don't trust them, there is not enough security, anyone can go on, can't they, really." (Louise)

Some parents regulated Internet use by physically being in the same room whilst websites were accessed. For example, one family used the Internet together:

"I find the sites for her telling her not to touch when popups come up, not to open them. If she wants to change the site, just give me a call and I'll change the site for her. So they're always under supervision." (Anthony father)

Other households, such as in the case of one family with two young children thought that it would be easier to monitor their children by also being physically present when consuming digital media content. In addition to this they have resorted to disconnecting the telephone line when not present, due to concerns about line charges as well as parental control.

"We have disconnected the telephone line cause if you have little ones and you are not careful you can end up running with massive bills” (Malcolm) 
With regards to TV, unbeknownst to parents, many teenagers knew the pin number needed to access restricted services. Two households with younger and older teens disconnected the telephone line from TV to prevent extra costs from the use of iTV functions.

Summing up our initial results, it is clear that participants have had several negative experiences with TV shopping, hidden costs, related to contacting customer services of TV shopping channels and additional costs related to quiz and game competitions. These result in increased security concerns and mistrust towards the iTV medium. In terms of parental control, parents prefer to monitor their children's media use by being present when a service or media is being consumed rather using specific parental control tools, which are seen as a not fully secure option. Lastly several participants expressed the view that use of profiles in the TV domain may help resolve privacy issues in a similar manner it does with PCs.

\section{THE EXPERIMENTAL DEVICE}

For the fourth and final visit to study participant's homes we developed an experimental home media device. The device functioned somewhat like a “technical probe”, as described by Hutchinson et al. [55], but crucially was only given to participants for the length of a single visit (30-60 minutes). The device was not the subject of a user interface (UI) or usability evaluation of any kind during this study, instead it was used as a focal point for the final visit, responding to and making concrete many of the ideas discussed with participants in the previous visits and encouraging participants to imagine how similar, fullyfunctional devices might function in the future. The focus of our research and this paper is not on the design and development of a portable second screen device, but rather on the concepts behind it. For this reason we are not offering a discussion on this. Readers who are interested in this subject may look at Cesar and Butlerman [30], [56] who have done extensive work in this field. 


\subsection{Our Design Response}

Based on participant feedback and concerns with security, parental control, hidden costs and privacy we developed a Pay-As-You-Go (PAYG) conceptual model enhanced with user profiles and incorporated aspects of this into the experimental device. The proposal of a PAYG payment model to pay for media services (iTV services, films, shopping) is a direct response to our findings that the possibility of unwittingly accumulating very large bills for interactive services is a strong disincentive for many iTV users, especially in households with children. Also by incorporating user profiles the PAYG concept becomes a more personalised experience catering for media consumption and financial transactions specific to individual members of a household.

Using the experimental device as a focal point, we generated discussion on whether and how households would employ similar devices in regulating spending, preventing unauthorised usage, parental controls over access to media and ways of making billing more transparent. The experimental device included a number of static screens illustrating these ideas (PAYG, Billing, user profiles), as well as fully interactive features that allowed participants to buy content, such as films and engage more with the PAYG concept (see Figure 1). We encouraged participants to explore the device, and to make use of the functional interactive features. Static screen illustrating more complex features such switching between profiles, purchasing credits and charging other profiles were included and shown to participants to prompt discussion and provide a feel for how such a system might work.

In this study we were more interested in participant responses to the ideas and concepts behind the experimental device rather than the device itself and user interface employed. Our main interest was in revealing households' opinions after making use of the device and in generating discussion as to where this could lead or be extended to, rather than focusing on the specifics of the device presented. 


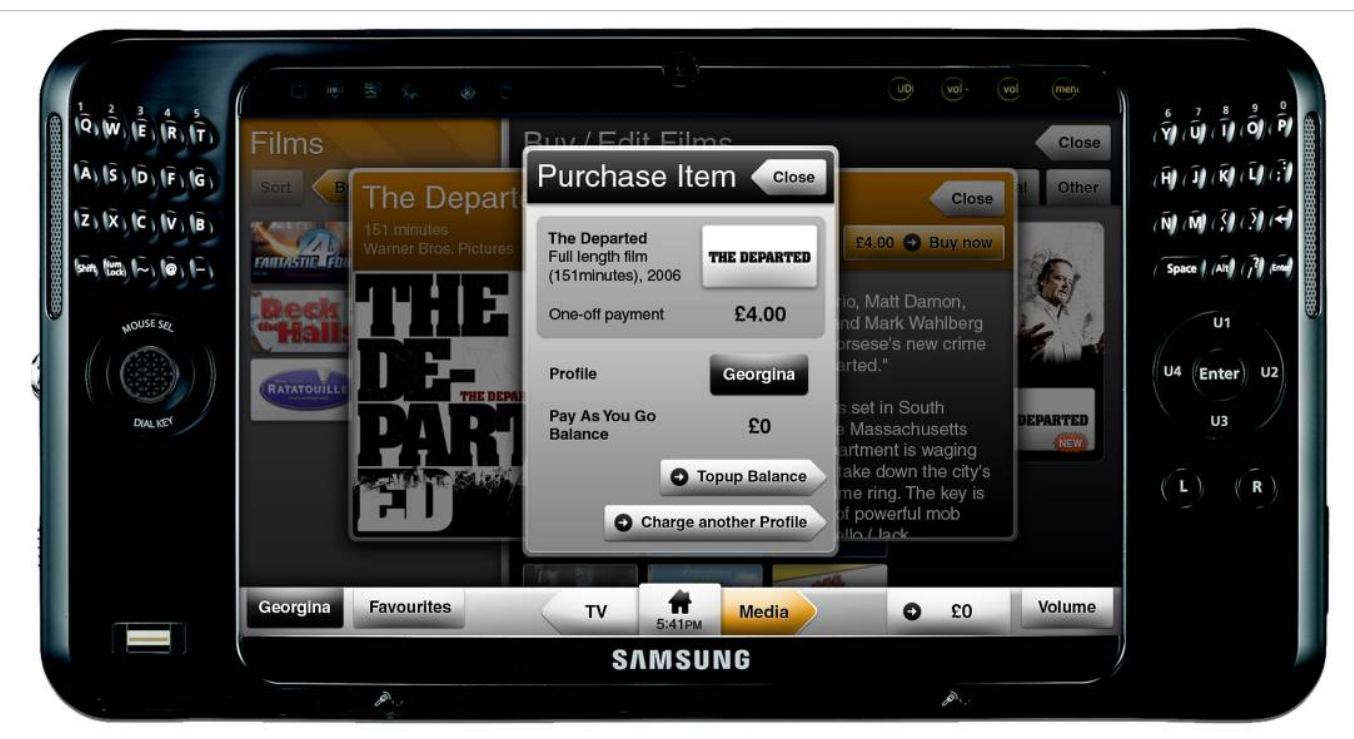

Fig 1 Purchasing a film using PAYG in the experimental device

\subsection{The Device}

The experimental device used in this study was designed to provide a realistic media-use experience for study participants in their own homes. The device connected wirelessly to a laptop, which was in turn connected to participant's TVs. This allowed actions performed on the experimental device to be instantly reflected on the TV within participant's normal viewing environment. The device provided various simulated functions, allowing control over the TV channel showing and allowed participant's to display photos on the TV screen.

The second screen device itself was created using a Samsung Q1 Ultra running Microsoft Windows XP. The device's dimensions were approximately 23 x $12 \mathrm{x}$ $23 \mathrm{~cm}$, with a 7 inch screen. The user interface displayed on the device was implemented using Adobe Flash connected wirelessly to a Java application running on a separate laptop, also running Microsoft Windows XP.

The UI itself was controlled entirely with the touch screen. The buttons visible to the right and left of the device's screen in figures 1, 2, 3 and 4 were not used in the study. Most actions were performed by touching regions of the screen.

Features of the UI specific to PAYG varied in terms of functionality. A significant part of the UI was given over allowing participants to select and organise content around their own preferences. This included purchasing content (in this case films) though the device. This portion of the system included pricing of items and dialogue messages asking users of the device to confirm the purchase 
of content. In addition to these fully-functional areas of the UI, we also added a number of static screens which showed mock-ups of the UI might appear during certain hypothetic actions, such as viewing a purchasing history or transferring credit between profiles on the device.

\section{$6 \quad$ Participant Responses}

Here we present some of the participant responses, suggestions and discussion generated as a result of using the experimental device to explore the PAYG concept. Participants indicated three main PAYG uses, namely regulating spending, preventing unauthorised use/spending by other household members and providing access to better billing information. Several participants commented on their familiarity with this concept in the mobile phone domain and the benefits of using a prepaid mode of financial transactions.

"Pay as you go, that's something people get used to now with phones and not only that you don't have something dictated to you when you pay as you go, you know there is options, there are choices so you don't feel as though you are being manipulated to a certain extent, if you want something buy it if no don't” (Jared)

Although the experimental device did not directly demonstrate PAYG for other interactive shopping transactions, we did use it to generate discussion around these possible uses for a similar device.

According to the data collected from our questionnaires, nine in ten households said they would use the PAYG to buy films and six out of ten said they would use it to pay for interactive services, such as voting. Five out of ten households said they would employ PAYG to buy goods through TV shopping channels.

When participants were asked whether they would like to top up their balance over the internet (using a normal PC) five out of ten responded yes, three out of ten no and two out of ten don't know. Interestingly when they were asked the same question but this time about toping their balance through their TV nine out of ten responded positively. This illustrates a strong majority trusting their TV more than the Internet when it comes to managing the PAYG account. 


\subsection{Regulating and Preventing Unauthorised Spending}

With regards to regulating spending, several participants highlighted the benefit a PAYG system would have for overall household finances if a budget were set for the family's entire media-related spending. There was also discussion on the need to have personal profiles. This was also reflected in the questionnaire data, where eight out of ten of the participants felt that different members of the family should have their own PAYG balance.

"so could you set a budget so you wouldn't go over a set amount say 20 pounds...and it would sort of come up like unavailable funds or whatever...it's nice to be able to see what you are using, that's a good idea I think, because sometimes you could get carried away” (Kelly)

Participants found this particularly useful as each household member would have his/her own profile to charge their media. Several households were willing to transfer credit to other members of the household/family using the PAYG service, with seven out of ten favouring this facility.

Other households, especially those with young children, emphasised how parents would employ this to set children spending allowances on a monthly basis to avoid excessive spending.

"if you had kids with their profile and they could have money in their topup, you could give them 10 pounds a month or something and they could decide how they would spend it and choose which films they would watch, yeah that would be a wicked idea" (Clara)

In some cases even children and teenagers favoured the use of PAYG by recognising the degree of independence that this would provide them with in their media purchases.

"If it was a Pay as you go thing I wouldn't have to hassle mom to ask whether I could watch this" (Peter)

Parents on the other hand highlighted the benefits of controlling costs, since once the initial top-up money value has been reached it cannot be exceeded or extended automatically as in the case of contract-based payments.

"iIf it is pay-as-you-go that's a good idea because if you top it up five founds or ten pounds you know if that runs out that's it" (Gary) 


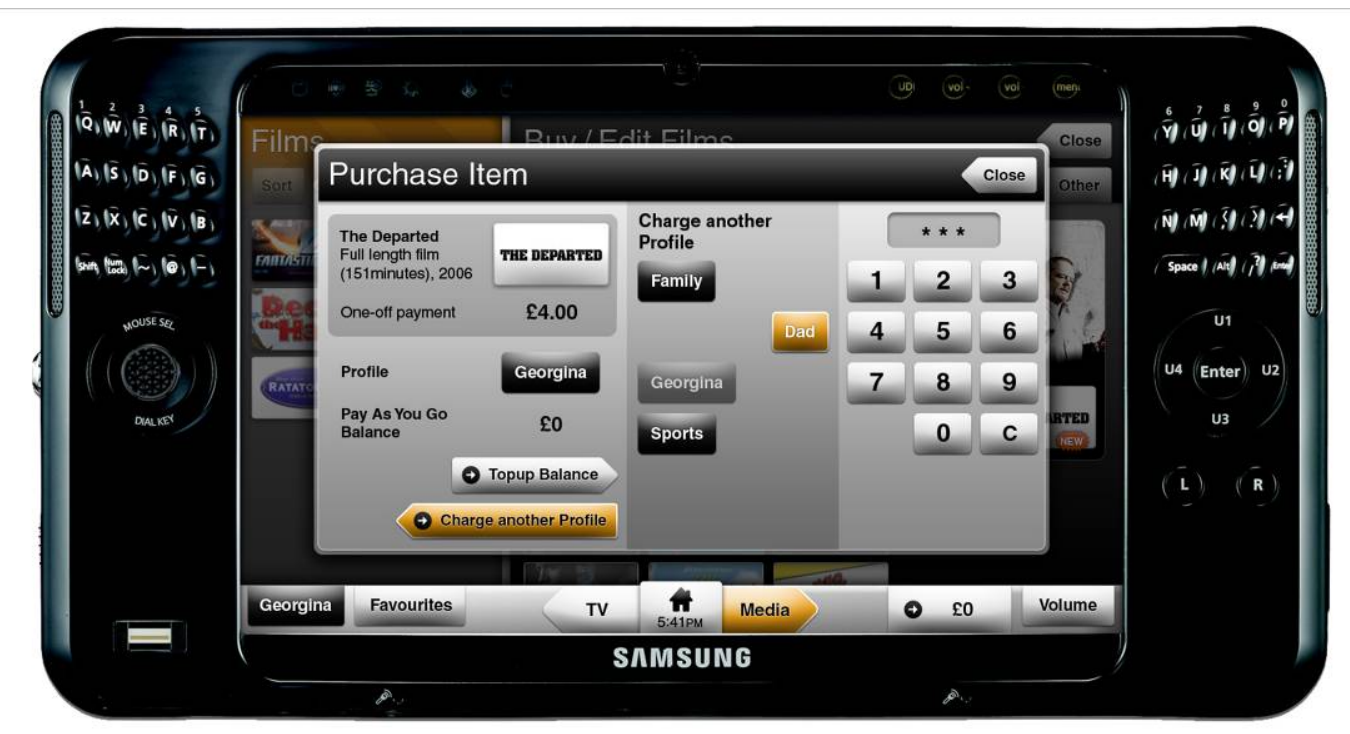

Fig. 2 Purchasing a film using PAYG by charging it on a different profile after pin authorisation

The PAYG concept was seen by several parents as a further safeguard to unauthorised spending, especially by younger members of the households (see Figure 2).

"if you are in a family environment you know that if your kid finds out your password they're only going to be able to spend so much money without costing without that constantly wrapping up" (Oprah)

They also indicated that it is useful for regulating the spending on rental movies order through the TV too, bringing control back to the parents.

"I've definitely think if you have young kids in the house and you want to have a limit on how many films they watch then you've got the control" (Mike)

Several participants suggested that PAYG is useful beyond family oriented households, applying equally to individuals sharing a house as well, indicating personal experiences and suggesting specific contexts to which this could be applied.

"for example in my brother's household where he owns the flat but he rents some rooms, this is quite good if people want to do that" (Tania)

On this topic, another participant shared his experience of unauthorised spending by with an extended family member suggesting that PAYG would have prevented this from happening. 
“cause I remember my other cousin came over and spent 2 weeks, I don't know what he was doing with remote, he ended up watching movies, I looked at the bill I said I never watched these movies" (Larry)

\subsection{Billing}

With regards to the process of the PAYG billing and reading statements, user responses were very encouraging. Nine out of ten households responded positively to the concept of viewing their PAYG statement on their TV using the experimental device (see Figure 3).

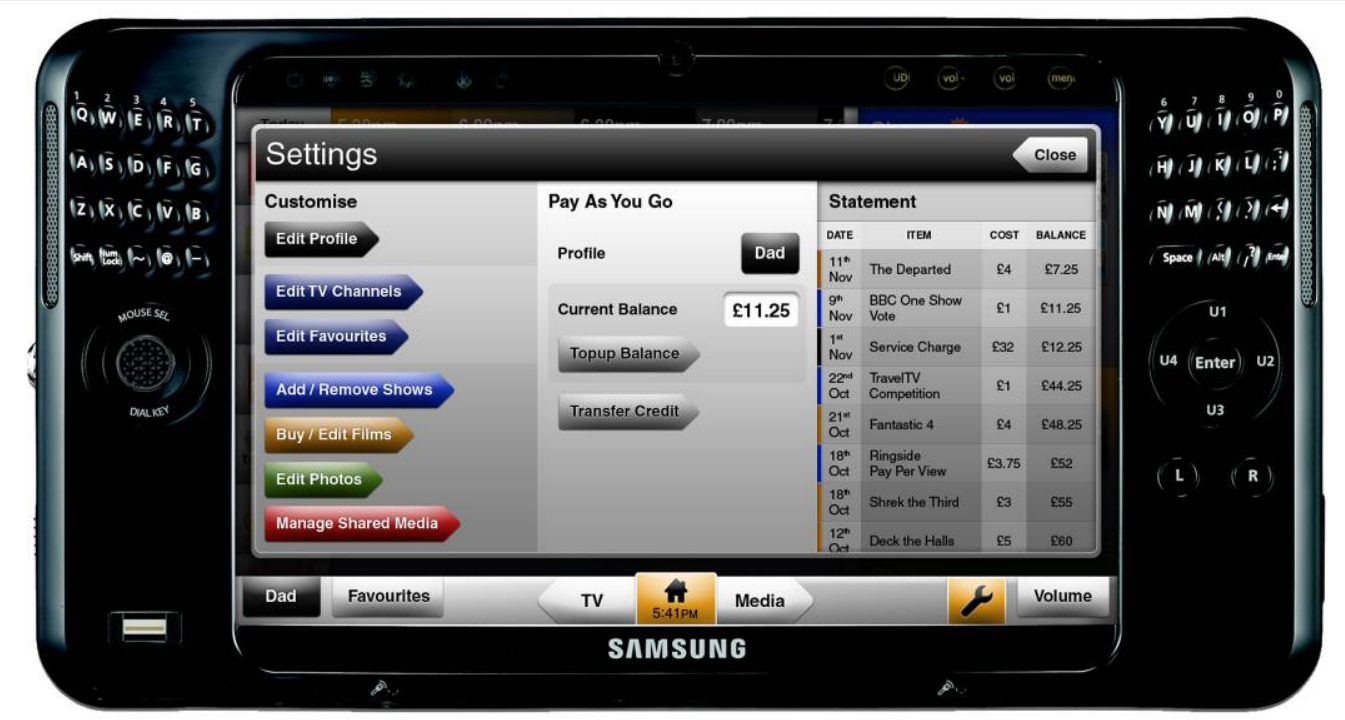

Fig. 3 Viewing the PAYG billing statement in the experimental device

When asked about the possibility of performing the same task on a PC, positive responses were limited to five out of ten households. The same preference was also observed in a question related to the toping up of the PAYG balance, where eight out of ten households replied they would do this using the experimental device, as opposed to five out of ten households willing to do this same activity over the using a PC. User responses were very encouraging with regards to the clarity and amount of information provided in the example billing statement, as well as the ability to constantly monitor the household's media related finances on how much is spent and where. 
"it's a good idea, cause then instead of like having to wait to the end of the month and realise that you've spent load and loads of money it's good to thing you can go in there you can see what you've bought and see how much you've got left" (Tracey)

Other participants commented on the information that is displayed during and after a transaction, to verify when and who in the household has purchased media.

"it tells you who the profile is, it tell you the date, how much it is and how much it's left, it's really good” (Connie)

It was also suggested that the PAYG concept should expand to allow the purchasing of other media, particularly sporting events and games, on a pay-perview basis.

"if you do the same thing with sporting events I suppose, where there's films if you've got box office like sky box office with the films you'd have a similar thing for sports” (Stewart)

\subsection{Parental Control}

During discussion, study participants suggested that two experimental devices, both synchronised to show which programmes were currently being watched, could be used to monitor their children's current viewing habits. This theme was not introduced as part of our study; instead it emerged in several households during discussion. Several parents suggested this novel parental control use of the experimental device as.

"if they've got TV in their room and they are watching at 12:00 and you want them to go to bed then you can see that they are watching and what they are watching” (Mark)

Parents emphasised that the experimental device could allow them to monitor what their children are viewing whilst not being physically in the same room as the TV.

"you can put it on a channel and then you know what channel they are watching and then if they did change it you can see what they've changed it to, if you are busy in the kitchen or something” (Carol) 


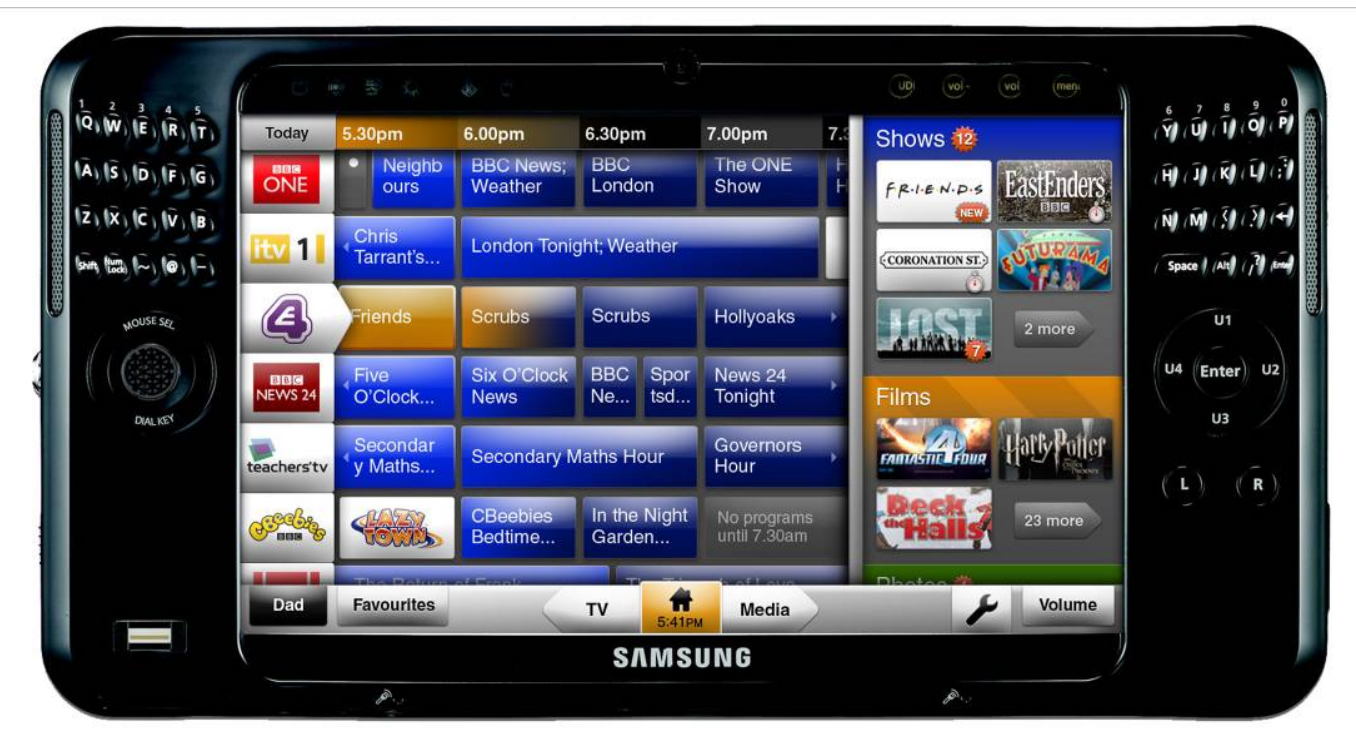

Fig. 4 The experimental device clearly indicating the TV channel and programme currently viewed by a household member (Scrubs on Channel 4 viewed by dad)

Some parents also expanded further of this use of the experimental device, suggesting that they can utilise it for monitoring children's spending on iTV contents, as this mother stated:

"you can see what they (children) are buying and how much they are spending, I think it's quite good idea." (Janet)

\section{CONCLUSION}

The paper has presented the results of an ethnographic study looking at trust, privacy and security issues associated with TV media in modern households. To aid the study an experimental touch screen device was introduced which simulated a number of new features for discussion with study participants. The experimental device proved a useful way of presenting new concepts and ideas, such as purchasing content, Pay-as-you-go (PAYG) billing and personalisation. Themes emerging from our research include negative feelings towards payment systems within iTV services and the desire for devices and services to incorporate the PAYG systems to help regulate spending and allow household members to manage their media purchases. With rapid expansion of marketplaces for digital content, these findings provide a valuable window into the issues iTV payment models have introduced for households. 
The need to control individual spending of the younger members in a household is contrasted and seems distinct from the desire for adult households members to control their own spending. Parents tended to want control over what their children had access to, both in terms of content and funds with which to purchase it. Likewise they want to shape their own access to content with self-imposed limits.

Negative experiences with iTV services and devices were commonly reported by participants during our study. Many of these negative experiences related directly to hidden costs and uncertainty about charges for services. PAYG offers a simple mechanism to control costs that is well known as a billing and payment model. As such PAYG provides method to control and shape purchases which is easy to understand. This, combined with ready access to purchase histories provides a means to address key barriers to iTV engagement and purchasing.

\section{REFERENCES}

[1] Seager W, Knoche H, Sasse M A. (2007) TV-centricity - Requirements gathering for triple play services. In Interactive TV: A Shared Experience. Proceedings of EuroITV 2007 May, Amsterdam, Netherlands, 274-8.

[2] Chorianopoulos K. (2008) User Interface Design Principles for Interactive Television Applications. Int J Hum Computer Interact. 24:6. 556 - 573. doi: 10.1080/10447310802205750

[3] Chorianopoulos K, Spinellis D, (2006) User interface evaluation of interactive TV: a media studies perspective. J Univers Access Inf Soc . 6:2. 209-218. doi: 10.1007/s10209-006-0032-1

[4] Bernhaupt R, Obrist M, Weiss A, Beck E, Tscheligi, E. (2008). Trends in the living room and beyond: results from ethnographic studies using creative and playful probing. ACM Comp. Entert. 6,:1. 1-23. doi: 10.1145/1350843.1350848

[5] Geerts D, Cesar P, Bulterman D. (2008) The implications of program genres for the design of social television systems. In Proceeding of the 1st international Conference on Designing interactive User Experiences for TV and Video. California, USA. October 2008, vol. 291. ACM, 71-80. Doi 10.1145/1453805.1453822

[6] Berglund A, Berglund E, Larsson A, and Bang A. (2006). Paper remote: an augmented television guide and remote control. J Univers. Access Inf. Soc. 4:4. 300-327. doi: 10.1007/s10209-004-0108-8

[7] Maglogiannis I, Makedon F, Pantziou G, and Baillie L. (2011). Pervasive technologies and assistive environments: social impact, financial, government and privacy issues. J Univers. Access Inf. Soc. 10:2. 111-114 doi: 10.1007/s10209-010-0200-1

[8] Gawlinski M. (2003). Interactive television production. Focal Press, Oxford 
[9] Pablo C, Dick C A Bulterman Janse J, Geerts D, Hendrik K, William S. (2009) Fragment, tag, enrich, and send: Enhancing social sharing of video. ACM Trans. Multimedia Comput. Commun. 5:3. 1-27. DOI=10.1145/1556134.1556136

[10] Carmichael A, Rice M, Sloan D, and Gregor P. (2006). Digital switchover or digital divide: a prognosis for usable and accessible interactive digital television in the UK. J Univers. Access Inf. Soc. 4: 4. 400-416. Doi: 10.1007/s10209-005-0004-X

[11] Dai X, Grundy J. (2007) NetPay: An off-line, decentralized micro-payment system for thinclient applications. Electron Commer Res Appl 6:1. 91-101. Doi: 10.1016/j.elerap.2005.10.009

[12] Chorianopoulos K. (2008) User Interface Design Principles for Interactive Television Applications. Int J Hum Computer Interact. 24:6. 556 - 573. doi: 10.1080/10447310802205750

[13] Chorianopoulos K, Spinellis D. (2006) User interface evaluation of interactive TV: a media studies perspective. J Univers. Access Inf. Soc. 6:2. 209-218. Doi: 10.1007/s10209-006-0032-1

[14] Cruickshank L, Tsekleves T, Whitham T, Hill A, Kondo K. (2007) Making interactive TV easier to use: Interface design for a second screen approach. The Design Journal. 10:3. 41-53 EISSN 1756-3062

[15] Baker K, Hulsen P. (2003) Multi-service models for interactive television. IEEE International Conference on Consumer Electronics. IEEE. 88 - 89. Doi: 10.1109/ICCE.2003.1218822

[16] England E, Finney A. (2002) Managing Multimedia: Project Management for Web and Convergent Media, Book 2: Technical Issues. Addison-Wesley, London

[17] Eronen L, Vuorimaa P. (2000) User Interfaces for Digital Television: a Navigator Case Study. Proceedings of conference on Advanced visual interfaces. In Proceedings of the working conference on Advanced visual interfaces (AVI '00). ACM, New York, USA, 276-279. DOI $=10.1145 / 345513.345346$.

[18] Tsekleves E, Whitham R, Kondo K, and Hill A. (2009). Bringing the television experience to other media in the home: an ethnographic study. In Proceedings of the seventh European conference on European interactive television conference (EuroITV '09). ACM, 201-210. DOI=10.1145/1542084.1542125

[19] Keeling K., Macaulay L A, McGoldrick, P J. (2007) DiTV and e-commerce among disadvantaged community groups. $\mathrm{J}$ of Behav Inf Technol 26:6. 545-560. Doi: 10.1080/01449290600709137

[20] Kaasinen E, Kulju M, Kivinen T, and Oksman V. (2009). User acceptance of mobile TV services. In Proceedings of the 11th international Conference on Human-Computer interaction with Mobile Devices and Services (Bonn, Germany, September 15 - 18, 2009). MobileHCI '09. ACM, 1-10. DOI =10.1145/1613858.1613902

[21] Holzinger A, Searle G, Wernbacher M. (2010). The effect of previous exposure to technology on acceptance and its importance in usability and accessibility engineering. J Univers. Access Inf. Soc. 1-16. Doi: 10.1007/s10209-010-0212

[22] Gauntlett D, Hill A. (1999) TV Living: Television, Culture and Everyday Life. Routledge, London.

[23] Inglesant P, Sasse A. (2007). Usability is the best policy: public policy and the lived experience of transport systems in London. In Proceedings of the 21st British CHI Group Annual 
Conference on HCI 2007: People and Computers Xxi: Hci..But Not As We Know It - Volume 1 British Computer Society, Swinton, UK, 35-44. ISBN: 978-1-902505-94-7

[24] Weisz D, Kiesler S, Zhang H, Ren Y, Kraut E, and Konstan A. (2007). Watching together: integrating text chat with video. In Proceedings of the SIGCHI Conference on Human Factors in Computing Systems (San Jose, California, USA, April 28 - May 03, 2007). CHI '07. ACM, 877886. DOI=10.1145/1240624.1240756

[25] Araujo I, Araujo Iv. (2003). Developing trust in internet commerce. InProceedings of the 2003 conference of the Centre for Advanced Studies on Collaborative research (CASCON '03). IBM Press 1-15.

[26] Lanford, P Hübscher, R. (2004) Trustworthiness in e-commerce, Proceedings of the 42nd annual Southeast regional conference, April 02-03, 2004 Alabama. ACM. 315 - 319. DOI=10.1145/986537.986614

[27] Patton M, Audun J. (2004). Technologies for Trust in Electronic Commerce. J Electron Commer Res. 4:1-2. 9-21. Doi: 10.1023/B:ELEC.0000009279.89570.27

[28] Buchinger S, Kriglstein S, Hlavacs H. (2009). A comprehensive view on user studies: survey and open issues for mobile TV. In Proceedings of the Seventh European Conference on European interactive Television Conference (Leuven, Belgium, June 03 - 05, 2009). EuroITV '09. ACM, 179-188. DOI=10.1145/1542084.1542121

[29] Bernhaupt R, Weiss A, Pirker M, WilfingerD, and Tscheligi M. (2010). Ethnographic insights on security, privacy, and personalization aspects of user interaction in interactive TV. In Proceedings of the 8th international interactive conference on Interactive TV and Video (EuroITV '10). ACM, USA, 187-196. DOI=10.1145/1809777.1809817

[30] Cesar P, Bulterman D, Jansen A. (2008) Usages of the Secondary Screen in an Interactive Television Environment: Control, Enrich, Share, and Transfer Television Content. In M. Tscheligi, M. Obrist, and A. Lugmayr (Eds.): EuroITV 2008, Springer-Verlag Berlin Heidelberg. 168-177. [31] Geerts D, De Grooff D. (2009). Supporting the social uses of television: sociability heuristics for social TV. In Proceedings of the 27th international Conference on Human Factors in Computing Systems (Boston, MA, USA, April 04 - 09, 2009). CHI '09. ACM, 595-604. DOI=10.1007/978-3-540-69478-6_22

[32] Williams D, UrsuF., Cesar P, Bergström K, Kegel I., and Meenowa J. (2009). An emergent role for TV in social communication. In Proceedings of the Seventh European Conference on European interactive Television Conference (Leuven, Belgium, June 03 - 05, 2009). EuroITV '09. ACM, 19-28. DOI=10.1145/1542084.1542088

[33] Cesar P, Chorianopoulos K. (2009). The Evolution of TV Systems, Content, and Users Toward Interactivity. Found. Trends Hum.-Comput. Interact. 2:4 373-95. Doi: $10.1561 / 1100000008$

[34] Semeraro G, Andersen V, Andersen H, Gemmis M, and Lops P. (2008). User profiling and virtual agents: a case study on e-commerce services. J Univers. Access Inf. Soc. 7:3. 179-194. Doi: 10.1007/s10209-008-0116-1

[35] Wilfinger D, Pirker M, Bernhaupt R, and Tscheligi M. (2009). Evaluating and investigating an iTV interaction concept in the field. In Proceedings of the Seventh European Conference on 
European interactive Television Conference (Leuven, Belgium, June 03 - 05, 2009). EuroITV '09. ACM, 175-178. DOI=10.1145/1542084.1542119

[36] Pirker M, Bernhaupt R, Mirlacher T. (2010). Investigating usability and user experience as possible entry barriers for touch interaction in the living room. InProceedings of the 8th international interactive conference on Interactive TV (EuroITV '10). ACM, 145-154. DOI=10.1145/1809777.1809808

[37] Vlachogiannis E, Gavalas D, Tsekouras G, and Anagnostopoulos C. (2010) Accessible interactive television using the MPEG-21 standard. J Univers. Access Inf. Soc. 10:2. Doi: 10.1007/s10209-010-0195-7

[38] Luyten K, Thys K, Huypens S, Coninx K. (2006). Telebuddies on the move: social stitching to enhance the networked gaming experience. In Proceedings of 5th ACM SIGCOMM Workshop on Network and System Support For Games (Singapore, October 30 - 31, 2006). NetGames '06. ACM, 18. DOI=10.1145/1230040.1230084

[39] Egelman S, Brush B, InkpenM. (2008). Family accounts: a new paradigm for user accounts within the home environment. In Proceedings of the ACM 2008 Conference on Computer Supported Cooperative Work (San Diego, CA, USA, November 08 - 12, 2008). CSCW '08. ACM, 669-678. DOI=10.1145/1460563.1460666

[40] Navarro-Prieto R, Rebaque-Rivas P, Hernandez-Pablo J. (2010). Recommending content for ITV: what the users really want?. In Proceedings of the 8th international interactive conference on Interactive TV (EuroITV '10). ACM, 123-126. DOI=10.1145/1809777.1809803

[41] O'Brien J, Rodden T, Rouncefield M, Hughes J. (1999). At home with the technology: an ethnographic study of a set-top-box trial. ACM Trans. Comput.-Hum. Interact. 6, 3 (Sep. 1999), 282-308. Doi: 10.1145/329693.329698

[42] Svoen, B. 2007. Consumers, Participants, and Creators: Young People's Diverse Use of Television and New Media. ACM Comput in Entertain. 5:2. 1-15. Doi: 10.1145/1279540.1279545 [43] Sauer G, Lazar J, Hochheiser H, and Feng J. (2010). Towards A Universally Usable Human Interaction Proof: Evaluation of Task Completion Strategies. ACM Trans. Access. Comput. 2:4, 132. Doi: $10.1145 / 1786774.1786776$

[44] Haines V, Mitchell V, Cooper C, Maguire M. (2007) Probing user values in the home environment within A technology driven Smart Home project. Pers. Ubiquit. Comput. 11, 349359. Doi: 10.1007/s00779-006-0075-6

[45] Lacohée H. Wakeford N. Pearson I. (2003) A Social History of the Mobile Telephone with a View of its Future, BT Technol J, 21:3. 203-211. Doi: 10.1023/A:1025187821567

[46] Patil V, Shyamasundar R (2005) e-coupons: An Efficient, Secure and Delegable MicroPayment System. Inf Syst Front. 7: 4-5. 371 - 389. Doi: 10.1007/s10796-005-4809-1

[47] Jorns O. Quirchmayr G. (2008). A middleware for location-based mobile applications with privacy protection. In Proceedings of the 10th international Conference on information integration and Web-Based Applications \& Services (Linz, Austria, November 24 - 26, 2008). G. Kotsis, D. Taniar, E. Pardede, and I. Khalil, Eds. iiWAS '08. ACM, 111-116. DOI=10.1145/1497308.1497333 
[48] Grinter R, Eldridge M. (2003) Wan2tlk?: Everyday text messaging. Proceedings of the SIGCHI Conference on Human Factors in Computing Systems. ACM Press. 441-448. DOI $=10.1145 / 642611.642688$

[49] Kurniawan S, Murni M, Yanuar, N. (2006). A Study of the Use of Mobile Phones by Older Persons. In CHI '06 extended abstracts on Human factors in computing systems (CHI EA '06). ACM, New York, NY, USA, 989-994. DOI=10.1145/1125451.1125641

[50] Hui P, Mortier R, Xu K, Crowcroft J, Li O. (2009) Sharing airtime with Shair avoids wasting time and money. In Proceedings of the 10th Workshop on Mobile Computing Systems and Applications (Santa Cruz, California, February 23 - 24, 2009). HotMobile '09. ACM, 1-6. DOI $=10.1145 / 1514411.1514417$

[51] Palankar R, Iamnitchi A, Ripeanu M, Garfinkel S. (2008). Amazon S3 for science grids: a viable solution?. In Proceedings of the 2008 international Workshop on Data-Aware Distributed Computing (Boston, MA, USA, June 24 - 24, 2008). DADC '08. ACM, 55-64. DOI=10.1145/1383519.1383526

[52] Bhattacharya J, Vashistha S. (2008. Utility computing-based framework for e-governance. In Proceedings of the 2nd international Conference on theory and Practice of Electronic Governance (Cairo, Egypt, December 01 - 04, 2008). T. Janowski and T. A. Pardo, Eds. ICEGOV '08, vol. 351. ACM, 303-309. DOI=10.1145/1509096.1509160

[53] Papa F, Livi S, Cornacchia M, Nicol E, Sapio B. (2010). Factors affecting the usage of payment services through digital television in Italy. In Proceedings of the 8th international interactive conference on Interactive TV (EuroITV '10). ACM, 209-216. DOI $=10.1145 / 1809777.1809820$

[54] Bocock, R. 1993. Consumption (Key Ideas). Routledge, London

[55] Hutchinson H, Mackay W, Westerlund B, et al. (2003) Technology probes: inspiring design for and with families. In Proceedings of the SIGCHI Conference on Human Factors in Computing Systems. CHI 2003. ACM. 17-24. DOI=10.1145/642611.642616

[56] Cesar P, Bulterman D, Jansen A.(2007) Social sharing of television content: An architecture. In: Proceedings of the IEEE Symposium on Multimedia (Workshops). 145-150. DOI=10.1109/ISMW.2007.80 